\title{
Identification of KRAS and PIK3CA but not BRAF mutations in patients with gastric cancer
}

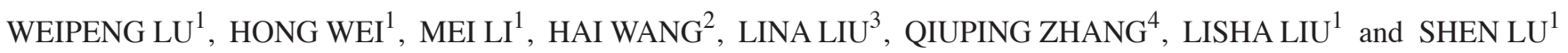 \\ ${ }^{1}$ Laboratory Center, The Second Affiliated Hospital of Dalian Medical University; ${ }^{2}$ Department of Pathology, \\ The Third People's Hospital of Dalian; Departments of ${ }^{3}$ Gastroenterology and ${ }^{4}$ Pathology, \\ The First Affiliated Hospital of Dalian Medical University, Dalian, Liaoning 116000, P.R. China
}

Received June 12, 2014; Accepted February 26, 2015

DOI: $10.3892 / \mathrm{mmr} .2015 .3530$

\begin{abstract}
Cetuximab, an immunoglobulin G1 chimeric monoclonal antibody directed against the epidermal growth factor receptor, is currently considered to be the strategy with the most potential for the treatment of gastric cancer due to the low frequency of KRAS mutations in patients with gastric cancer. However, the therapeutic success of cetuximab in colorectal cancer (CRC) has demonstrated that the clinical effect of cetuximab is closely dependent not only on KRAS mutations, but also BRAF and phosphoinositide-3-kinase, catalytic, $\alpha$ polypeptide (PIK3CA) mutations. In the present study, the status of KRAS, BRAF and PIK3CA mutations in gastric cancer were investigated concomitantly in order to aid the selection of patients eligible for treatment with cetuximab. Mutations in KRAS (exon 2), BRAF (exon 15) and PIK3CA (exon 9 and exon 20) were retrospectively evaluated by high resolution melting analysis and DNA direct sequencing in samples from 156 patients with gastric cancer. Mutations in either KRAS or PIK3CA were identified in 13 samples (8.3\%), 7 samples with KRAS mutations and 6 samples with PIK3CA mutations. No mutations in the BRAF gene were identified. The frequency of mutations in either KRAS or PIK3CA were significantly higher in patients without lymph node metastasis than those with. Furthermore, KRAS and PIK3CA mutations were mutually exclusive. The present study, therefore, suggested that it may be necessary to evaluate KRAS and PIK3CA mutations concomitantly for the selection of patients eligible for treatment with cetuximab.
\end{abstract}

Correspondence to: Professor Shen Lu, Laboratory Center, The Second Affiliated Hospital of Dalian Medical University, 467 Zhongshan Road, Dalian, Liaoning 116000, P.R. China

E-mail: lv.weipeng@aliyun.com

Key words: KRAS, phosphoinositide-3-kinase, catalytic, $\alpha$ polypeptide, BRAF, mutation, cetuximab, gastric cancer

\section{Introduction}

Gastric cancer is one of the most common types of cancer globally, with a particularly high incidence in Northeast Asian countries, including China, Japan and Korea (1). The traditional therapeutic strategies for gastric cancer have failed to effectively treat the condition (2). In previous years, targeted therapies against the molecular variants specific to the carcinogenesis of various types of human cancer have been developed. However, the development of targeted therapies for gastric cancer lags behind other types of cancer. Trastuzumab, a monoclonal antibody against human epidermal growth factor receptor 2 (HER2) widely used for the treatment of breast cancer, has been approved for the treatment of patients with gastric cancer expressing HER2 based on the encouraging results of Trastuzumab for the treatment of gastric cancer (3). Gefitinib, an epidermal growth factor receptor-tyrosine kinase inhibitor (EGFR-TKI) successfully used for the treatment of non-small cell lung cancer with an EGFR mutation in exon 19 or 21, appears to be effective for certain patients with gastric cancer (4). Furthermore, EGFR mutations in exon 21 have been identified in patients with gastric cancer by a Portuguese research group and in our previous study $(5,6)$. These findings indicate that certain patients with gastric cancer would benefit from TKIs. However, only a specific subpopulation of patients are able to benefit from treatment with trastuzumab or EGFR-TKIs

Cetuximab, an immunoglobulin G1 chimeric monoclonal antibody directed against EGFR, exhibits anticancer effects by binding to the extracellular domain of EGFR with a higher affinity than the natural ligands of the receptor, inhibiting its dimerization and phosphorylation, and then blocking the downstream signaling transduction pathways, mainly KRAS/BRAF/mitogen activated protein kinase and phosphoinositide 3-kinase (PI3K)/AKT/mammalian target of rapamycin (7). Cetuximab was initially approved for the treatment of patients with colorectal cancer (CRC), particularly those with EGFR-positive tissue samples. Notably, it was reported that over half of all patients with gastric cancer expressed EGFR, suggesting that certain patients with gastric cancer may benefit from cetuximab treatment (8). Furthermore, cetuximab, either alone or in combination with various chemotherapeutic drugs, has been evaluated in clinical trials for the 
treatment of patients with gastric cancer (9-11). However, the majority of patients, including those with EGFR-positive tissue samples were not sensitive to cetuximab. The previous results appear to be conflicting. Therefore, it is hypothesized that certain factors may impair the sensitivity of patients with gastric cancer to cetuximab. Currently, cetuximab has been well demonstrated to be effective in patients with CRC without KRAS mutations as mutant KRAS with an elevated kinase activity would result in a continuously activated state, leading to a continuous and self-sufficient signaling transduction pathway, thus rendering cetuximab ineffective (12). Furthermore, it has been clearly demonstrated that mutations of other downstream effectors of EGFR, such as BRAF and PIK3CA, are also able to render cetuximab ineffective $(13,14)$. Therefore, the evaluation of KRAS, BRAF and PIK3CA mutations in gastric cancer may aid the selection of patients eligible for treatment with cetuximab.

In previous years, KRAS mutations have been reported in gastric cancer with a frequency, ranging between 0 and $21 \%$ (15-18). However, the exact frequency of mutations in the KRAS gene and their association with clinicopathological features of the patients remains to be elucidated. Although previous studies had considered BRAF mutations to be rare in gastric cancer, further studies are required to confirm this hypothesis as no more than ten studies have investigated the status of the BRAF mutation to date, to the best of our knowledge (18-25). In contrast to KRAS and BRAF mutations, a mutation in PIK3CA has been identified in gastric cancer with a relatively higher frequency between 4.3 and $25.0 \%$ (18,26-28). However, no definitive conclusion can be drawn regarding whether the PIK3CA mutation coexists with the KRAS mutation in gastric cancer.

Therefore, the previously mentioned data revealed that mutations in KRAS, BRAF and PIK3CA may be involved in gastric cancer. In addition, these variants were usually evaluated by different groups from different countries and data regarding comprehensive analysis of KRAS, BRAF and PIK3CA mutations in gastric cancer were limited. The aim of the present study was to evaluate concomitantly the status of KRAS, BRAF and PIK3CA mutations in 156 gastric cancer samples in order to aid the selection of patients eligible for treatment with cetuximab.

\section{Patients and methods}

Study population. A total of 156 gastric cancer samples [53 fresh-frozen and 103 formalin-fixed, paraffin-embedded (FFPE)] were collected from patients who underwent surgical resection at The First Affiliated Hospital of Dalian Medical University and The Third People's Hospital of Dalian (Dalian, China). The present study was initiated with the approval of the ethics committee of The Second Affiliated Hospital of Dalian Medical University. Informed consent was obtained from patients prior to the collection of the samples. None of patients in the present study had received preoperative radio- or chemotherapy. The clinicopathological features of all patients were verified by two experienced pathologists (Table I).

DNA extraction. The DNA extraction of the 53 fresh-frozen samples has been previously completed by our group (17).
Therefore, in the present study, DNA was only extracted from the 103 FFPF samples. An $\sim 5-\mu$ m thick single section from each sample was selected for hematoxylin and eosin (H\&E) staining. An experienced pathologist analyzed the H\&E sections and marked the area containing at least $70 \%$ tumor cells. Subsequently, according to the marked area in the H\&E section, the tumor tissue was dissected from eight sections of each FFPE sample and then genomic DNA was extracted using an FFPE DNA extraction kit (Omega Bio-Tek, Inc., Norcross, GA, USA) according to the manufacturer's instructions.

Mutation analysis of KRAS (exon 2), BRAF (exon 15) and PIK3CA (exon 9 and 20). The status of the KRAS (exon 2) mutation in the 53 fresh-frozen samples has been previously analyzed and reported by Liu et al (17). In the present study, the mutation status of KRAS (exon 2) in 103 FFPE tumor samples, and BRAF (exon 15) and PIK3CA (exon 9 and 20) in 156 tumor samples was screened using polymerase chain reaction-high resolution melting (PCR-HRM) analysis. PCR primers for KRAS, BRAF and PIK3CA are shown in Table II. The final PCR reaction mixture $(10 \mu \mathrm{l})$ contained: i) KRAS exon 2: 1X PCR buffer, $200 \mu \mathrm{M}$ dNTPs, $2.5 \mathrm{mM} \mathrm{MgCl}_{2}, 1 \mu \mathrm{M}$ each primer, 0.25 units HotStart Taq (Takara Bio Inc., Dalian, China), 5 ng genomic DNA and 1X LC Green Plus (Biofire Diagnostics, Salt Lake City, UT, USA); ii) BRAF exon 15 and PIK3CA exon 9: 1X PCR Buffer, $200 \mu \mathrm{M} \mathrm{dNTPs,} 2 \mathrm{mM} \mathrm{MgCl}_{2}$, $1 \mu \mathrm{M}$ each primer, 0.25 units HotStart Taq, 5 ng genomic DNA and 1X LC Green Plus; iii) PIK3CA exon 20: 1X PCR Buffer, $200 \mu \mathrm{M}$ dNTPs, $2.5 \mathrm{mM} \mathrm{MgCl}_{2}, 0.5 \mu \mathrm{M}$ each primer, 0.25 units HotStart Taq, 5 ng genomic DNA and $1 \mathrm{X}$ LC Green Plus. The PCR amplification conditions were as follows: i) KRAS exon 2: $95^{\circ} \mathrm{C}$ for $10 \mathrm{~min} ; 45$ cycles of $95^{\circ} \mathrm{C}$ for $30 \mathrm{sec}, 54^{\circ} \mathrm{C}$ for $10 \mathrm{sec}$ and $72^{\circ} \mathrm{C}$ for $1 \mathrm{~min}$; ii) BRAF exon 15 and PIK3CA exon 20: $95^{\circ} \mathrm{C}$ for $10 \mathrm{~min} ; 45$ cycles of $95^{\circ} \mathrm{C}$ for $30 \mathrm{sec}, 56^{\circ} \mathrm{C}$ for $10 \mathrm{sec}$ and $72^{\circ} \mathrm{C}$ for $30 \mathrm{sec}$; and iii) PIK3CA exon 9: $95^{\circ} \mathrm{C}$ for $10 \mathrm{~min}$; 45 cycles of $95^{\circ} \mathrm{C}$ for $30 \mathrm{sec}, 60^{\circ} \mathrm{C}$ for $10 \mathrm{sec}$ and $72^{\circ} \mathrm{C}$ for $30 \mathrm{sec}$.

DNA sequencing. The PCR product was purified using the GeneJET $^{\mathrm{TM}}$ gel extraction kit (Thermo Fisher Scientific, Rockford, IL, USA) according to the manufacturer's instructions and then sequenced on an ABI Prism 3730 sequence detection system (Takara Bio, Inc.). A 173 bp amplicon was generated by sequencing primers of KRAS exon 2 following the same method as the PCR-HRM primers above. A 124 bp amplicon was generated by sequencing primers of PIK3CA exon 9, forward: 5'-GTAACAGACTAGCTAGAG-3' and reverse: 5'-CTGTGACTCCATAGAAAATC-3'. A 158 bp amplicon was generated by sequencing primers of PIK3CA exon 20, forward: 5'-GAATGCCAGAACTACAATC-3' and reverse: 5'-TGTGTGGAAGATCCAATC-3'.

The sequencing conditions for KRAS and PIK3CA were as follows: $95^{\circ} \mathrm{C}$ for $10 \mathrm{~min} ; 45$ cycles of $95^{\circ} \mathrm{C}$ for $30 \mathrm{sec}, 54^{\circ} \mathrm{C}$ for $10 \mathrm{sec}$ and $72^{\circ} \mathrm{C}$ for $1 \mathrm{~min}$.

Statistical analysis. All statistical analyses were conducted using the $\chi^{2}$ test and Fisher's exact test with SPSS 13.0 statistical software (SPSS, Inc., Chicago, IL, USA). P $<0.05$ was considered to indicate a statistically significant difference. 
Table I. Associations between gene mutations and clinicopathological features of patients.

\begin{tabular}{|c|c|c|c|c|c|c|c|}
\hline \multirow[b]{2}{*}{ Clinicopathological feature } & \multirow[b]{2}{*}{ No. } & \multicolumn{2}{|c|}{ KRAS } & \multicolumn{2}{|c|}{ PIK3CA } & \multicolumn{2}{|c|}{ KRAS/PIK3CA } \\
\hline & & Mutation & P-value & Mutation & $\mathrm{P}$-value & Mutation & P-value \\
\hline Gender & & & 1 & & 1 & & 0.956 \\
\hline Male & 115 & 5 & & 4 & & 9 & \\
\hline Female & 41 & 2 & & 2 & & 4 & \\
\hline Age (years) & & & 0.439 & & 1 & & 0.385 \\
\hline$\leq 65$ & 78 & 2 & & 3 & & 5 & \\
\hline$>65$ & 78 & 5 & & 3 & & 8 & \\
\hline Lymph node metastasis & & & 0.131 & & 0.359 & & 0.036 \\
\hline Present & 116 & 3 & & 3 & & 6 & \\
\hline Absent & 40 & 4 & & 3 & & 7 & \\
\hline Differentiation grade & & & $0.742^{\mathrm{a}}$ & & $0.53^{\mathrm{a}}$ & & $0.317^{\mathrm{a}}$ \\
\hline Well & 5 & 1 & & 1 & & 2 & \\
\hline Moderate & 42 & 2 & & 2 & & 4 & \\
\hline Poor & 92 & 4 & & 3 & & 7 & \\
\hline MAC & 17 & 0 & & 0 & & 0 & \\
\hline Depth of invasion & & & 0.433 & & 1 & & 0.296 \\
\hline $\mathrm{T} 1$ & 4 & 1 & & 0 & & 1 & \\
\hline $\mathrm{T} 2-\mathrm{T} 4$ & 152 & 6 & & 6 & & 12 & \\
\hline
\end{tabular}

${ }^{a}$ Well and Moderate vs. Poor and MAC. MAC, mucinous adenocarcinoma. PIK3CA, phosphoinositide-3-kinase, catalytic, $\alpha$ polypeptide.

Table II. Primers for mutation analysis using polymerase chain reaction-high resolution melting.

\begin{tabular}{ll}
\hline Primer & \multicolumn{1}{c}{ Sequence (from 5' to 3') } \\
\hline KRAS exon 2 & $\begin{array}{l}\text { Forward: AGGCCTGCTGAAAATGACTG } \\
\text { Reverse: TCAAAGAATGGTCCTGCACC }\end{array}$ \\
BRAF exon 15 & $\begin{array}{l}\text { Forward: CTCTTCATAATGCTTGCTCTGATAGG } \\
\text { Reverse: TAGTAACTCAGCAGCATCTCAGG }\end{array}$ \\
PIK3CA exon 9 & Forward: CTAGCTAGAGACAATGAATTAAGGGAAA \\
PIK3CA exon 20 & Reverse: CATTTTAGCACTTACCTGTGACTCCA \\
& Forward: TGAGCAAGAGGCTTTGGAGT \\
& Reverse: TCATTTTCTCAGTTATCTTTTCAGTTCAAT
\end{tabular}

PIK3CA, phosphoinositide-3-kinase, catalytic, $\alpha$ polypeptide.

\section{Results}

A total of 156 gastric cancer samples were screened for mutations in KRAS (exon 2), BRAF (exon 15) and PIK3CA (exon 9 and 20). As a whole, 8.3\% (13/156) of the analyzed samples harbored a mutation in either KRAS or PIK3CA. No mutations in the BRAF gene were identified. Notably, the frequency of mutations in either KRAS or PIK3CA was significantly higher in patients without lymph node metastasis than those with (17.5 vs. $5.2 \%, \mathrm{P}=0.036)$. No significant association was observed between mutations in either KRAS or PIK3CA and other clinicopathological features (Table I).
KRAS mutations. Mutations in the KRAS gene were identified in 7 out of 156 gastric cancer samples (4.5\%). In addition to the five mutations (two G13D, one G12V and two G12D) previously described by Liu et al (17), a further two mutations were found to occur at codon 12, leading to the substitutions of glycine for aspartic acid (G12D) and alanine (G12A), respectively (Fig. 1). In addition, the two mutations occurred exclusively in female patients aged over 65 years whose tumors were histologically classified into poorly-differentiated adenocarcinoma without lymph node metastasis. The mutation types and the clinicopathological features of the patients are summarized in Table III. The results demonstrated that no significant associa- 


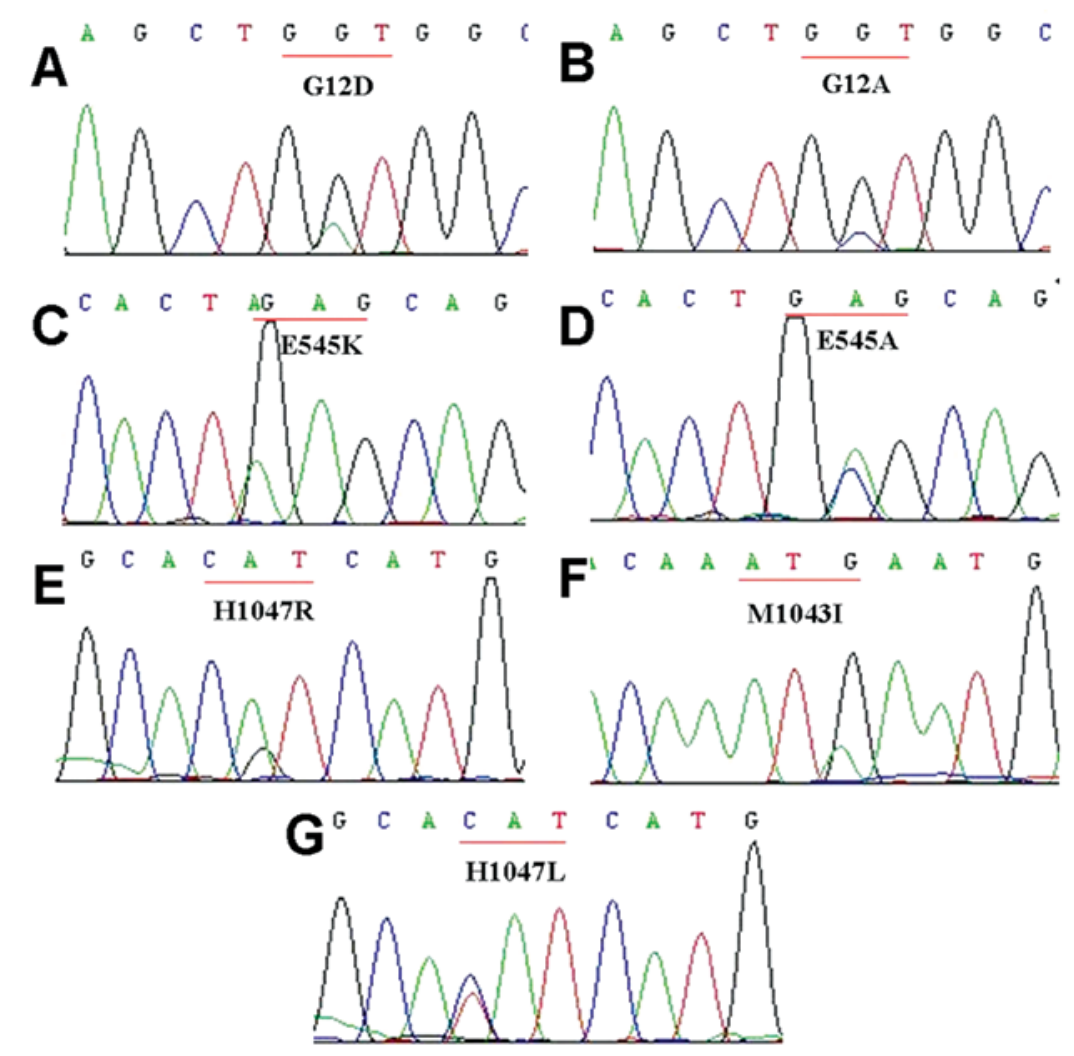

Figure 1. Sequencing chromatograms of (A and B) KRAS exon 2,(C and D) PIK3CA exon 9, and (E, F and G) PIK3CA exon 20 mutations. PIK3CA, phosphoinositide-3-kinase, catalytic, $\alpha$ polypeptide.

tion was found between mutations in the KRAS gene and the clinicopathological features of the patients (Table I).

BRAF mutations. Mutations in the BRAF gene were not detected in the present study, which is consistent with the results of previous studies.

PIK3CA mutations. Mutations in the PIK3CA gene were identified in 6 out of 156 gastric cancer samples (3.8\%), including three mutations in exon 9 (two E545K and one E545A) and four mutations in exon 20 (two H1047R, one M1043I and one H1047L) (Fig. 1). Furthermore, two different types of PIK3CA mutations, namely E545A in exon 9 and H1047L in exon 20, occurred simultaneously in one patient with gastric cancer. By contrast, mutations in the PIK3CA gene were found to occur in a mutually exclusive manner with mutations in the KRAS gene. The mutation types and the clinicopathological features of the patients are summarized in Table III. In addition, no significant association was found between mutations in the PIK3CA gene and the clinicopathological features of the patients (Table I).

\section{Discussion}

In the previous decade, molecular targeted therapy has been an important area of investigation for the treatment of human cancer. However, contemporary targeted therapy for gastric cancer is less than satisfactory, with few encouraging therapeutic effects. In addition to trastuzumab, which has been approved for the treatment of patients with gastric cancer, gefitinib appears to be useful clinically. It is worth noting that trastuzumab and gefitinib are applicable to only a small faction of patients with gastric cancer. Cetuximab, which was initially implemented for the treatment of patients with $\mathrm{CRC}$, is currently undergoing clinical trials for the treatment of patients with gastric cancer. However, clinical outcomes from certain trials have been unsuccessful $(9,10)$. In CRC, cetuximab has been well demonstrated to be effective in patients without mutations in the KRAS gene (12). However, certain patients without mutations in the KRAS gene do not respond to cetuximab in clinical practice, suggesting that other molecular variants may be associated with the inefficacy of cetuximab. Various studies have demonstrated that patients with CRC carrying mutations of other downstream effectors of EGFR, such as BRAF and PIK3CA, also exhibit resistance to treatment with cetuximab $(13,14)$. Therefore, a previous study suggested that patients with CRC without KRAS, BRAF and PIK3CA mutations are more likely to respond to cetuximab (29). Based on the therapeutic success of cetuximab in $\mathrm{CRC}$, it was hypothesized that it is necessary to evaluate the status of KRAS, BRAF and PIK3CA mutations in gastric cancer for selecting patients eligible for treatment with cetuximab.

KRAS, a molecular switch of intracellular signaling transduction pathways, is essential in transferring extracellular growth signals into the nucleus. Mutant KRAS protein exhibits elevated kinase activity and is able to constitutively activate downstream signaling transduction pathways independent of stimuli from activated EGFRs. A review conducted by Kiaris and Spandidos (30) demonstrated that 
Table III. Mutation types and clinicopathological features of the patients.

\begin{tabular}{|c|c|c|c|c|c|c|c|}
\hline \multirow{2}{*}{$\begin{array}{l}\text { Patient } \\
\text { no. }\end{array}$} & \multirow[b]{2}{*}{ KRAS } & \multicolumn{2}{|c|}{ PIK3CA } & \multirow[b]{2}{*}{ Gender } & \multirow{2}{*}{$\begin{array}{c}\text { Age } \\
\text { (years) }\end{array}$} & \multirow{2}{*}{$\begin{array}{l}\text { Differentiation } \\
\text { grade }\end{array}$} & \multirow{2}{*}{$\begin{array}{c}\text { Lymph node } \\
\text { metastasis }\end{array}$} \\
\hline & & Exon 9 & Exon 20 & & & & \\
\hline 1 & G13D & & & Male & 61 & Well & Absent \\
\hline 9 & G12D & & & Male & 72 & Moderate & Present \\
\hline 27 & G12V & & & Male & 64 & Poor & Present \\
\hline 29 & G12D & & & Male & 70 & Poor & Present \\
\hline 49 & G13D & & & Male & 73 & Moderate & Absent \\
\hline 68 & G12D & & & Female & 69 & Poor & Absent \\
\hline 152 & G12A & & & Female & 75 & Poor & Absent \\
\hline 39 & & & H1047R & Female & 81 & Moderate & Absent \\
\hline 45 & & E545K & & Male & 65 & Moderate & Present \\
\hline 70 & & & H1047R & Female & 73 & Well & Absent \\
\hline 83 & & & M1043I & Male & 45 & Poor & Absent \\
\hline 108 & & E545K & & Male & 61 & Poor & Present \\
\hline 122 & & E545A & H1047L & Male & 67 & Poor & Present \\
\hline
\end{tabular}

PIK3CA, phosphoinositide-3-kinase, catalytic, $\alpha$ polypeptide.

a mutation in the KRAS gene was associated with numerous types of human cancer, including pancreatic cancer, CRC and lung cancer (30). To date, a wide variety of studies have investigated the status of mutations in the KRAS gene in gastric adenocarcinoma with the frequency ranging between 0 and 21\% (15-18). The frequency of mutations in the KRAS gene in different studies has varied markedly. In order to improve understanding of the status of mutations in the KRAS gene in gastric cancer, a large international multi-center study, including 712 patients with gastric cancer was conducted in 2013 (31). In the study, which is the largest to date, the overall frequency of mutations in the KRAS gene was $4 \%$ and KRAS mutation was not associated with clinicopathological features, including ethnicity, gender and stage of tumor differentiation. The discrepancy between previous studies and the multi-center study may be predominantly due to the small sample size. Thus far, the majority of the studies included $\leq 100$ patients with gastric cancer, rendering the interpretation of any conclusion difficult. In our previous study (17), the frequency of mutations in the KRAS gene was 9.6\%. Furthermore, the patients with KRAS mutations were exclusively males although no significant difference was found, due to the small sample size. However, in the current study with a larger sample size, it was identified that the frequency of mutations in the KRAS gene was $4.5 \%$. In addition, the patients with mutations in the KRAS gene were not only males but also females. No significant association was identified between KRAS mutation and the clinicopathological features of the patient, including gender, age, differentiation grade and depth of invasion. Thus, mutations in the KRAS gene may occur in all patients with gastric cancer, rendering its evaluation necessary for the identification of patients suitable for cetuximab therapy.

BRAF, a member of the RAF kinase family, is an essential downstream effector of KRAS. BRAF is commonly activated by somatic mutation and mutated BRAF is able to constitu- tively activate downstream signaling transduction pathways regardless of stimulus from EGFR or KRAS. To date, BRAF mutations have been identified in numerous types of human cancer, including melanoma, papillary thyroid cancer and CRC (32-34). However, data regarding the BRAF mutation in gastric cancer are limited. To date, a small number of studies involving 943 patients with gastric cancer have investigated the status of the BRAF mutation and found that the average frequency of mutations in the BRAF gene was only $2.5 \%$ (ranging between 0 and 11\%) (18-25). Therefore, it is not noteworthy that no mutations in the BRAF gene were found in the present study. Mutations in the BRAF gene were absent or occurred only occasionally in Chinese patients with gastric cancer. Therefore, it is not necessary to evaluate the status of the BRAF mutation in order to select patients eligible for treatment with cetuximab.

PI3K, another downstream effector of EGFR, usually interacts with KRAS in the regulation of cellular functions. PIK3CA, encoding the catalytic subunit of PI3K, was initially demonstrated to be mutated in human cancer by Samuels et al (27). Subsequently, PIK3CA mutations have been identified in various types of human cancer, including breast cancer, CRC, liver cancer and ovarian cancer $(28,35,36)$. In gastric cancer, the frequency of PIK3CA mutations varied between 4.3 and $25 \%(18,26-28)$. In the present study, the frequency of PIK3CA mutations was $3.8 \%$, slightly lower than that observed in previous studies. The discrepancy among these studies may be due to a number of reasons, including sample size, mutation detection method used and the ethnicity of the patients. In CRC, it has been reported that PIK3CA mutations usually coexist with KRAS mutations $(28,37)$. Notably, the PIK3CA mutation was also observed in a concomitant manner with KRAS mutation in gastric cancer $(26,28)$. However, in the present study, it was identified that PIK3CA and KRAS mutations were mutually exclusive. Therefore, a 
definitive conclusion regarding the association of the PIK3CA mutation with the KRAS mutation in gastric cancer requires confirmation in further studies. By contrast, it was identified that mutations in either KRAS or PIK3CA were more likely to occur in patients without lymph node metastasis, which indicated that mutations of downstream effectors of EGFR occurred earlier in gastric carcinogenesis. In any case, KRAS and PIK3CA mutations should be evaluated prior to patients receiving cetuximab.

In conclusion, in the present study $\sim 8.3 \%$ of Chinese patients with gastric cancer harbored a mutation in either KRAS or PIK3CA, suggesting that these patients would not benefit from cetuximab. Therefore, in the future, KRAS and PIK3CA, but not BRAF mutations should be evaluated in order to select patients eligible for treatment with cetuximab.

\section{Acknowledgements}

The authors would like to thank the doctors of the Second Affiliated Hospital of Dalian Medical University for the collection of the samples. This study was supported by the National Natural Science Foundation of China (grant no. 81071805).

\section{References}

1. Jemal A, Bray F, Center MM, Ferlay J, Ward E and Forman D: Global cancer statistics. CA Cancer J Clin 61: 69-90, 2011.

2. Janunger KG, Hafström L, Nygren P, Glimelius B; SBU-group. Swedish Council of Technology Assessment in Health Care: A systematic overview of chemotherapy effects in gastric cancer. Acta Oncol 40: 309-326, 2001.

3. Bang YJ, Van Cutsem E, Feyereislova A, et al: Trastuzumab in combination with chemotherapy versus chemotherapy alone for treatment of HER2-positive advanced gastric or gastro-oesophageal junction cancer (ToGA): a phase 3 , open-label, randomised controlled trial. Lancet 376: 687-697, 2010.

4. Rojo F, Tabernero J, Albanell J, et al: Pharmacodynamic studies of gefitinib in tumor biopsy specimens from patients with advanced gastric carcinoma. J Clin Oncol 24: 4309-4316, 2006.

5. Moutinho C, Mateus AR, Milanezi F, Carneiro F, Seruca R and Suriano G: Epidermal growth factor receptor structural alterations in gastric cancer. BMC Cancer 8: 10, 2008.

6. Liu Z, Liu L, Li M, et al: Epidermal growth factor receptor mutation in gastric cancer. Pathology 43: 234-238, 2011.

7. Ciardiello $\mathrm{F}$ and Tortora G: EGFR antagonists in cancer treatment. N Engl J Med 358: 1160-1174, 2008.

8. Rivera F, Vega-Villegas ME and López-Brea MF: Cetuximab, its clinical use and future perspectives. Anticancer Drugs 19: 99-113, 2008.

9. Chan JA, Blaszkowsky LS, Enzinger PC, et al: A multicenter phase II trial of single-agent cetuximab in advanced esophageal and gastric adenocarcinoma. Ann Oncol 22: 1367-1373, 2011.

10. Schoennemann KR, Bjerregaard JK, Hansen TP, et al: Biweekly cetuximab and irinotecan as second-line therapy in patients with gastro-esophageal cancer previously treated with platinum. Gastric Cancer 14: 219-225, 2011.

11. Shi M, Ji J, Wu J, et al: Cetuximab combined with FOLFOX4 as the first-line treatment for advanced gastric cancer: report of 25 cases from a single institution. Hepatogastroenterology 59: 1054-1058, 2012

12. Karapetis CS, Khambata-Ford S, Jonker DJ, et al: K-ras mutations and benefit from cetuximab in advanced colorectal cancer. N Engl J Med 359: 1757-1765, 2008.

13. De Roock W, Claes B, Bernasconi D, et al: Effects of KRAS, BRAF, NRAS and PIK3CA mutations on the efficacy of cetuximab plus chemotherapy in chemotherapy-refractory metastatic colorectal cancer: a retrospective consortium analysis. Lancet Oncol 11: 753-762, 2010.
14. Sartore-Bianchi A, Di Nicolantonio F, Nichelatti M, et al: Multi-determinants analysis of molecular alterations for predicting clinical benefit to EGFR-targeted monoclonal antibodies in colorectal cancer. PLoS One 4: e7287, 2009.

15. Victor T, Du Toit R, Jordaan AM, Bester AJ and van Helden PD: No evidence for point mutations in codons 12,13 and 61 of the ras gene in a high-incidence area for esophageal and gastric cancers. Cancer Res 50: 4911-4914, 1990.

16. Hongyo T, Buzard GS, Palli D, et al: Mutations of the K-ras and p53 genes in gastric adenocarcinomas from a high-incidence region around Florence, Italy. Cancer Res 55: 2665-2672, 1995.

17. Liu ZM, Liu LN, Li M, Zhang QP, Cheng SH and Lu S: Mutation detection of KRAS by high-resolution melting analysis in Chinese gastric cancer. Oncol Rep 22: 515-520, 2009.

18. Corso G, Velho S, Paredes J, et al: Oncogenic mutations in gastric cancer with microsatellite instability. Eur J Cancer 47: 443-451, 2011.

19. Lee SH, Lee JW, Soung YH, et al: BRAF and KRAS mutations in stomach cancer. Oncogene 22: 6942-6945, 2003.

20. Kim IJ, Park JH, Kang HC, et al: Mutational analysis of BRAF and K-ras in gastric cancers: absence of BRAF mutations in gastric cancers. Hum Genet 114: 118-120, 2003.

21. Oliveira C, Pinto M, Duval A, et al: BRAF mutations characterize colon but not gastric cancer with mismatch repair deficiency. Oncogene 22: 9192-9196, 2003.

22. Wu M, Semba S, Oue N, Ikehara N, Yasui W and Yokozaki H: BRAF/K-ras mutation, microsatellite instability and promoter hypermethylation of hMLH1/MGMT in human gastric carcinomas. Gastric Cancer 7: 246-253, 2004.

23. Zhao W, Chan TL, Chu KM, Chan AS, Stratton MR, Yuen ST and Leung SY: Mutations of BRAF and KRAS in gastric cancer and their association with microsatellite instability. Int J Cancer 108: 167-169, 2004.

24. Sasao S, Hiyama T, Tanaka S, Yoshihara M, Yasui W and Chayama K: Clinicopathologic and genetic characteristics of gastric cancer in young male and female patients. Oncol Rep 16: 11-15, 2006.

25. Stella G, Rojas Llimpe F, Barone C, et al: KRAS and BRAF mutational status as response biomarkers to cetuximab combination therapy in advanced gastric cancer patients. ASCO Meet Abstr 27: e15503, 2009.

26. Li VS, Wong CW, Chan TL, et al: Mutations of PIK3CA in gastric adenocarcinoma. BMC Cancer 5: 29, 2005.

27. Samuels Y, Wang Z, Bardelli A, et al: High frequency of mutations of the PIK3CA gene in human cancers. Science 304: $554,2004$.

28. Velho S, Oliveira C, Ferreira A, et al: The prevalence of PIK3CA mutations in gastric and colon cancer. Eur J Cancer 41: 1649-1654, 2005

29. Bardelli A and Siena S: Molecular mechanisms of resistance to cetuximab and panitumumab in colorectal cancer. J Clin Oncol 28: 1254-1261, 2010.

30. Kiaris H and Spandidos D: Mutations of ras genes in human tumors (review). Int J Oncol 7: 413-421, 1995.

31. van Grieken NC, Aoyama T, Chambers PA, et al: KRAS and BRAF mutations are rare and related to DNA mismatch repair deficiency in gastric cancer from the East and the West: Results from a large international multicentre study. Br J Cancer 108: 1495-1501, 2013.

32. Davies H, Bignell GR, Cox C, et al: Mutations of the BRAF gene in human cancer. Nature 417: 949-954, 2002.

33. Lee SH, Ahn BK, Baek SU and Chang HK: BRAF mutation in multiple primary cancer with colorectal cancer and stomach cancer. Gastroenterol Rep (Oxf) 1: 70-74, 2013.

34. Henke LE, Perkins SM, Pfeifer JD, Ma C, Chen Y, DeWees T and Grigsby PW: BRAF V600E mutational status in pediatric thyroid cancer. Pediatr Blood Cancer 61: 1168-1172, 2014.

35. Campbell IG, Russell SE, Choong DY, et al: Mutation of the PIK3CA gene in ovarian and breast cancer. Cancer Res 64: 7678-7681, 2004

36. Lee JW, Soung YH, Kim SY, et al: PIK3CA gene is frequently mutated in breast carcinomas and hepatocellular carcinomas. Oncogene 24: 1477-1480, 2005.

37. Simi L, Pratesi N, Vignoli M, et al: High-resolution melting analysis for rapid detection of KRAS, BRAF and PIK3CA gene mutations in colorectal cancer. Am J Clin Pathol 130: 247-253, 2008. 